\title{
Validation of Left Ventricular Ejection Fraction with the IQ•SPECT System in Small-Heart Patients
}

Hiroto Yoneyama ${ }^{1}$, Takayuki Shibutani ${ }^{2}$, Takahiro Konishi ${ }^{1}$, Asuka Mizutani ${ }^{1}$, Ryosuke Hashimoto ${ }^{2}$, Masahisa Onoguchi ${ }^{2}$, Koichi Okuda ${ }^{3}$, Shinro Matsuo ${ }^{4}$, Kenichi Nakajima ${ }^{4}$, and Seigo Kinuya ${ }^{4}$

${ }^{I}$ Department of Radiological Technology, Kanazawa University Hospital, Kanazawa, Japan; ${ }^{2}$ Department of Health Science, Graduate School of Medical Science, Kanazawa University, Kanazawa, Japan; ${ }^{3}$ Department of Physics, Kanazawa Medical University, Kanazawa, Japan; and ${ }^{4}$ Department of Nuclear Medicine, Kanazawa University Hospital, Kanazawa, Japan

The IQ·SPECT system, which is equipped with multifocal collimators (SMARTZOOM) and uses ordered-subset conjugate gradient minimization as the reconstruction algorithm, reduces the acquisition time of myocardial perfusion imaging compared with conventional SPECT systems equipped with low-energy high-resolution collimators. We compared the IQ•SPECT system with a conventional SPECT system for estimating left ventricular ejection fraction (LVEF) in patients with a small heart (end-systolic volume $<20 \mathrm{~mL}$ ). Methods: The study consisted of 98 consecutive patients who underwent a 1-d stress-rest myocardial perfusion imaging study with a ${ }^{99 m}$ Tc-labeled agent for preoperative risk assessment. Data were reconstructed using filtered backprojection for conventional SPECT and ordered-subset conjugate gradient minimization for IQ•SPECT. End-systolic volume, end-diastolic volume, and LVEF were calculated using quantitative gated SPECT (QGS) and cardioREPO software. We compared the LVEF from gated myocardial perfusion SPECT to that from echocardiographic measurements. Results: End-diastolic volume, end-systolic volume, and LVEF as obtained from conventional SPECT, IQ•SPECT, and echocardiography showed a good to excellent correlation regardless of whether they were calculated using QGS or using cardioREPO. Although LVEF calculated using QGS significantly differed between conventional SPECT and IQ-SPECT $(65.4 \% \pm 13.8 \%$ vs. $68.4 \% \pm 15.2 \%)(P=0.0002)$, LVEF calculated using cardioREPO did not $(69.5 \% \pm 10.6 \%$ vs. $69.5 \% \pm 11.0 \%)$. Likewise, although LVEF calculated using QGS significantly differed between conventional SPECT and IQ.SPECT (75.0 \pm 9.6 vs. $79.5 \pm 8.3)(P=0.0005)$, LVEF calculated using cardioREPO did not $(72.3 \% \pm 9.0 \%$ vs. $74.3 \% \pm 8.3 \%)$. Conclusion: In small-heart patients, the difference in LVEF between $\mathrm{IQ} \cdot$ SPECT and conventional SPECT was less when calculated using cardioREPO than when calculated using QGS.

Key Words: IQ-SPECT; echocardiographic measurement; cardioREPO; small heart; left ventricular ejection fraction

J Nucl Med Technol 2017; 45:201-207

DOI: $10.2967 /$ jnmt.116.188680

\footnotetext{
Received Dec. 22, 2016; revision accepted May 9, 2017.

For correspondence or reprints contact: Hiroto Yoneyama, Department of Radiological Technology, Kanazawa University Hospital, 13-1 Takara-machi, Kanazawa 920-8641, Japan.

E-mail: kizu@cf6.so-net.ne.jp

Published online Jun. 13, 2017.

COPYRIGHT (c) 2017 by the Society of Nuclear Medicine and Molecular Imaging.
}

$\mathbf{T}$ he IQ•SPECT system (Siemens) is equipped with dedicated multifocal collimators that have a cardiocentric orbit (SMARTZOOM; Siemens) and uses an advanced reconstruction algorithm optimized for their special geometry (ordered-subset conjugate gradient minimization) (1-4). These features enable a reduced acquisition time and a distancedependent implementation that makes 3-dimensional resolution recovery possible (5).

Gated myocardial perfusion SPECT, although used to evaluate left ventricular ejection fraction (LVEF), is not accurate for this purpose in patients with a small heart (6-10). Many researchers have reported that measurement of LVEF is more accurate with the IQ-SPECT system than with conventional SPECT systems that use low-energy high-resolution collimators (11-14). However, few studies have focused on patients with a very small left ventricular volume, as is frequently observed in Japanese women. Recently, a software program (EXINI Heart [EXINI Diagnostics], marketed in Japan as cardioREPO [FUJIFILM RI Pharma Co. Ltd.]) included an algorithm to compensate for the small-heart effect - that is, the systematic overestimation of LVEF in small-heart patients (6). In this study, a small heart was defined as one with an endsystolic volume (ESV) of less than $20 \mathrm{~mL}$ as calculated using quantitative gated SPECT software (QGS; Cedars Sinai Medical Center). The purpose of this study was to compare the LVEF from IQ-SPECT to that from conventional SPECT when calculated using QGS and cardioREPO.

\section{MATERIALS AND METHODS}

\section{Patient Population}

The study comprised 98 consecutive patients, 40 of whom had a small heart (Table 1). All patients underwent myocardial perfusion imaging using a 1-d protocol for preoperative risk assessment. For the stress study, 250-370 MBq of ${ }^{99 \mathrm{~m} T c-s e s t a m i b i}$ (FUJIFILM RI Pharma Co. Ltd.) or ${ }^{99 m}$ Tc-tetrofosmin (Nihon Medi-Physics Co., Ltd.) were injected after an adenosine stress test; for the rest study, the injection was 740-1,110 MBq. The patients underwent SPECT imaging approximately $60 \mathrm{~min}$ after the injection. Sixty cases were initially evaluated with IQ•SPECT and then with conventional SPECT. The remaining 38 cases were initially examined with conventional SPECT and then with IQ•SPECT. The average myocardial counts per pixel were measured from a $45^{\circ}$ left anterior 
TABLE 1

Patient Characteristics

\begin{tabular}{|c|c|}
\hline Characteristic & Finding \\
\hline Mean age $\pm S D(y)$ & $72.5 \pm 9.4$ (range, $28-88)$ \\
\hline \multicolumn{2}{|l|}{$\operatorname{Sex}(n)$} \\
\hline Male & $77(78.6 \%)$ \\
\hline Female & $21(21.4 \%)$ \\
\hline Mean body mass index \pm SD & $\begin{array}{l}23.5 \pm 3.1 \\
\text { (range, 16.4-32.8) }\end{array}$ \\
\hline Mean body height \pm SD $(\mathrm{cm})$ & $\begin{array}{l}161.1 \pm 9.4 \\
\quad \text { (range, 134-189) }\end{array}$ \\
\hline Mean body weight \pm SD $(\mathrm{kg})$ & $\begin{array}{l}61.7 \pm 11.3 \\
\quad(\text { range, } 37-93.2)\end{array}$ \\
\hline \multicolumn{2}{|l|}{ Tracer $(n)$} \\
\hline Sestamibi & $2(2 \%)$ \\
\hline Tetrofosmin & 96 (98\%) \\
\hline \multicolumn{2}{|l|}{ Main risk factors $(n)$} \\
\hline $\begin{array}{l}\text { Obesity (body mass } \\
\text { index }>25 \text { ) }\end{array}$ & $30(30.6 \%)$ \\
\hline Diabetes mellitus & $10(10.2 \%)$ \\
\hline Abdominal aortic aneurysm & $37(37.8 \%)$ \\
\hline Thoracic aortic aneurysm & $24(24.5 \%)$ \\
\hline Angina pectoris & $10(10.2 \%)$ \\
\hline Arteriosclerosis obliterans & $9(9.2 \%)$ \\
\hline $\begin{array}{l}\text { Previous coronary artery } \\
\text { bypass surgery }\end{array}$ & $5(5.1 \%)$ \\
\hline Aortic dissection & $5(5.1 \%)$ \\
\hline Common iliac artery aneurysm & $3(3.1 \%)$ \\
\hline $\begin{array}{l}\text { Abnormal electrocardiographic } \\
\text { pattern }\end{array}$ & $2(2.0 \%)$ \\
\hline Renal artery aneurysm & $1(1.0 \%)$ \\
\hline Hepatic artery aneurysm & $1(1.0 \%)$ \\
\hline Aortic valve stenosis & $1(1.0 \%)$ \\
\hline
\end{tabular}

oblique projection. The studies were electrocardiogram-gated at 16 frames per R-R interval. Automated analysis was performed on the gated SPECT images to calculate end-diastolic volume (EDV), ESV, and LVEF, via QGS and cardioREPO. Myocardial perfusion defects were analyzed semiquantitatively by a 17 -segment model with 5-point visual scoring. The uptake in each segment was measured; left ventricular function was analyzed using quantitative perfusion SPECT software (QPS, Cedars Sinai Medical Center). The study was approved by the institutional ethics committee, and written informed consent was obtained from all patients before they participated.

\section{Conventional SPECT Acquisition and Reconstruction}

The acquisition and reconstruction parameters commonly used for IQ•SPECT and conventional SPECT are shown in Table 2. The conventional system was a Symbia T6 SPECT/CT scanner (Siemens), which consisted of a dual-head $\gamma$-camera in a $180^{\circ}$ geometry, equipped with a low-energy high-resolution collimator. SPECT data were acquired in a $64 \times 64$ matrix for a total of 20 min, using $\times 1.45$ zooming, $6.6-\mathrm{mm}$ pixels, and $35 \mathrm{~s}$ per projection. In total, 60 frames were obtained over $360^{\circ}$, at $6^{\circ}$ per frame. The cardiac cycle was divided into 16 frames for electrocardiogram gating. $\mathrm{R}-\mathrm{R}$ interval, with an average of $\pm 25 \%$ on the electrocardiogram monitor, was accepted for gating. The energy window was set to $15 \%$ (centered on $140 \mathrm{keV}$ ). Scatter and attenuation correction were not used. The detectors were placed near the patient in circular mode with a radius of $24-25 \mathrm{~cm}$, and data were reconstructed using filtered backprojection without resolution correction (e.soft application package; Siemens). An eighthorder Butterworth filter was used for filtered backprojection, with a cutoff of 0.39 cycles/cm for QGS and 0.52 cycles/cm for QPS.

\section{IQ·SPECT Acquisition and Reconstruction}

IQ•SPECT was performed using a dual-head SPECT/CT system. The projection datasets were acquired over $208^{\circ}$ cardiocentric orbits with 17 views per detector for $9 \mathrm{~s}$, resulting in a total acquisition time of $5 \mathrm{~min}$. A cardiac cycle was divided into 16 frames for electrocardiogram gating. R-R interval, with an average of $\pm 25 \%$ on the electrocardiogram monitor, was accepted for gating. The energy window was set to $15 \%$ (centered on $140 \mathrm{keV}$ ). Rotation of the detectors was circular, with a $28-\mathrm{cm}$ radius centered on the heart. The SPECT images were reconstructed using ordered-subset conjugate gradient minimization with 30 iterations and 1 subset for QGS and 10 iterations and 3 subsets for QPS. The reconstructed images were

TABLE 2

Common SPECT Acquisition and Reconstruction Parameters

\begin{tabular}{|c|c|c|}
\hline Parameter & Conventional SPECT & IQ·SPECT \\
\hline Reconstruction algorithm & Filtered backprojection & OSCGM \\
\hline Collimator & LEHR & SMARTZOOM \\
\hline Energy window & $140 \mathrm{keV} \pm 7.5 \%$ & $140 \mathrm{keV} \pm 7.5 \%$ \\
\hline Number of projections & 60 views (30 per detector) & 34 views (17 per detector) \\
\hline Rotation range & $360^{\circ}$ & $208^{\circ}$ \\
\hline Acquisition time (min) & 20 (35 s per projection) & 5 (9 s per projection) \\
\hline Magnification & $\times 1.45$ & $\times 1.00$ \\
\hline Rotation radius (cm) & 24-25 & 28 \\
\hline Number of iterations & & QGS: 30, QPS: 10 \\
\hline Number of subsets & & QGS: 1, QPS: 3 \\
\hline Updates & & 30 \\
\hline Gaussian filter (mm) & & 9.6 \\
\hline Butterworth filter (cycles/cm) & QGS: 0.39 , QPS: 0.52 & \\
\hline Matrix & $64 \times 64$ & $128 \times 128$ \\
\hline Pixel size (mm) & 6.6 & 4.8 \\
\hline
\end{tabular}


postsmoothed using a 3-dimensional gaussian spatial filter (full width at half maximum [FWHM], $9.6 \mathrm{~mm}$ ); CT-based attenuation correction and scatter correction were used on the IQ•SPECT data. The CT acquisitions were obtained on the 6-slice CT scanner of the Symbia T6 system. CT was performed using a tube voltage of $130 \mathrm{kVp}$, a tube current-time exposure of $20 \mathrm{~mA} \cdot \mathrm{s}$ (product of tube current and time), a slice thickness of $5 \mathrm{~mm}$, and a B08s reconstruction kernel. Images were also reconstructed without attenuation and scatter correction. Patient motion during an acquisition was manually corrected.

\section{Echocardiography}

M-mode, 2-dimensional echocardiography was used to measure left ventricular volume. The maximum minor axes of the left ventricle at end-diastole and end-systole were measured on the parasternal long-axis view, assuming a spheroid shape. Left ventricular volume was usually calculated using the formula of Teichholz et al. (15):

$$
\mathrm{V}=7.0 \times \mathrm{D}^{3} /(2.4+\mathrm{D})
$$

where $\mathrm{D}$ is the dimension of the ventricle.

\section{Compensation for the Small-Heart Effect}

The new method for delineating the left ventricle, cardioREPO, is based on a method that has been previously described in detail $(16,17)$. Briefly, the heart-shaped left ventricular model was adjusted in an iterative process to optimize the fit of the midmyocardial surface to the 3-dimensional image data of the first frame and then to subsequent frames separately without constraints regarding left ventricular basal wall motion. The endocardial and epicardial surfaces were defined symmetrically as the position corresponding to $75 \%$ of the maximal pixel count along each sampling profile perpendicular to the left ventricular wall. The left ventricular volume was calculated using the endocardial surface and the valve plane. No manual corrections were applied for left ventricular delineation by cardioREPO. In patients with a small heart, endocardial and epicardial edge tracing becomes inappropriate because of blurring of the myocardial border. To avoid such effects, the method was additionally modified and adjusted for small hearts by shifting the endocardial and epicardial surfaces in the epicardial direction before left ventricular volume calculations (Fig. 1). The contour was corrected automatically. The size of the shift was calculated using the nongated midventricular volume of the left ventricle and a second-degree univariate polynomial equation. The processing resulted in a maximum shift of $3.5 \mathrm{~mm}$ at a midventricular volume of $0 \mathrm{~mL}$ and decreased the shift up to a volume of $85 \mathrm{~mL}$, for which the shift was $0 \mathrm{~mm}$.

\section{Statistical Analysis}

Linear regression and Bland-Altman analysis were performed among the echocardiography, conventional SPECT, and IQ•SPECT images using the least-squares method. The Student $t$ test was used for paired data. Statistical significance was defined as a $P$ level of less than 0.05. Statistical analysis was performed using GraphPad Prism, version 5.0 (GraphPad Software), for Windows (Microsoft).

\section{RESULTS}

Using conventional SPECT, myocardial count density was $133.9 \pm 38.0$ and $269.1 \pm 74.3$ counts per pixel for the stress and rest tests, respectively. Additionally, patients who underwent stress and rest tests in conjunction with IQ•SPECT

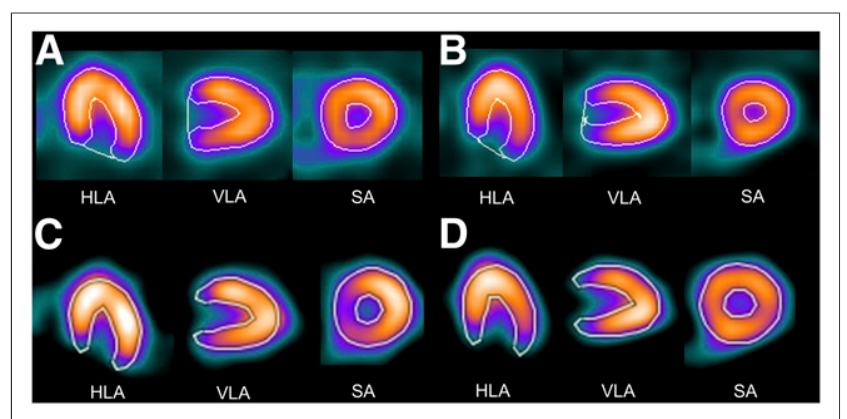

FIGURE 1. Gated myocardial perfusion SPECT images of 67-yold woman for whom echocardiographic measurements were $110 \mathrm{~mL}$ for ESV, $29 \mathrm{~mL}$ for EDV, and 73\% for LVEF. (A) Conventional SPECT with QGS: EDV, $60 \mathrm{~mL}$; ESV, $9 \mathrm{~mL}$; and LVEF, 86\%. (B) IQ·SPECT with QGS: EDV, $49 \mathrm{~mL}$; ESV, $4 \mathrm{~mL}$; and LVEF, 92\%. (C) Conventional SPECT with cardioREPO: EDV, $71 \mathrm{~mL}$; ESV, $12 \mathrm{~mL}$; and LVEF, 84\%. (D) IQ.SPECT with cardioREPO: EDV, $70 \mathrm{~mL}$; ESV, $15 \mathrm{~mL}$; and LVEF, 78\%. HLA = horizontal long axis; $\mathrm{SA}=$ short axis; VLA = vertical long axis.

had myocardial counts of $15.1 \pm 4.5$ and $30.0 \pm 8.2$ per pixel, respectively. Figure 2 shows that counts/s in the myocardium were approximately 4 times higher with IQ SPECT than with conventional SPECT.

EDV, ESV, and LVEF as calculated using either QGS or cardioREPO had a good to excellent correlation among echocardiography, conventional SPECT, and IQ•SPECT (Table 3). Bland-Altman plots for LVEF are shown in Figure 3. The gradients of the linear regression lines in the plots were -0.104 for conventional SPECT with QGS versus IQ•SPECT with QGS, 0.296 for conventional SPECT with QGS versus echocardiography, 0.410 for IQ•SPECT with QGS vs. echocardiography, -0.039 for conventional SPECT with cardioREPO vs. IQ•SPECT with cardioREPO, -0.026 for conventional SPECT with cardioREPO versus echocardiography, and 0.017 for IQ•SPECT with cardioREPO vs. echocardiography. Although the overall results for LVEF significantly differed between conventional

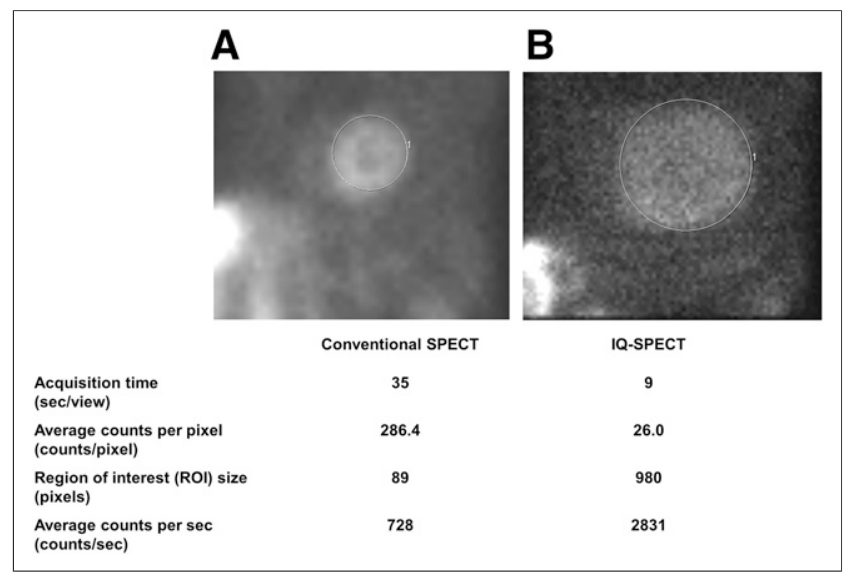

FIGURE 2. Average myocardial counts per pixel as measured from $45^{\circ}$ left anterior oblique projection on conventional SPECT (A) and IQ.SPECT (B). 
TABLE 3

Comparison of EDV, ESV, and LVEF for Conventional SPECT, IQ•SPECT, and Echocardiography

\begin{tabular}{|c|c|c|c|c|c|c|}
\hline Parameter & Conventional & IQ & Echo & Conventional vs. IQ & Conventional vs. echo & IQ vs. echo \\
\hline \multicolumn{7}{|l|}{ QGS } \\
\hline $\mathrm{EDV}(\mathrm{mL})$ & $77.0 \pm 39.1$ & $74.2 \pm 36.8$ & $110.6 \pm 38.3$ & $r=0.98$ & $r=0.77$ & $r=0.77$ \\
\hline $\mathrm{ESV}(\mathrm{mL})$ & $30.0 \pm 29.2$ & $27.4 \pm 27.3$ & $40.6 \pm 26.7$ & $r=0.97$ & $r=0.81$ & $r=0.82$ \\
\hline LVEF (\%) & $65.4 \pm 13.8$ & $68.4 \pm 15.2$ & $64.8 \pm 11.6$ & $r=0.86$ & $r=0.63$ & $r=0.64$ \\
\hline \multicolumn{7}{|l|}{ CardioREPO } \\
\hline $\mathrm{EDV}(\mathrm{mL})$ & $87.9 \pm 35.6$ & $82.8 \pm 34.0$ & $110.6 \pm 38.3$ & $r=0.98$ & $r=0.77$ & $r=0.75$ \\
\hline ESV (mL) & $28.1 \pm 22.6$ & $27.0 \pm 22.4$ & $40.6 \pm 26.7$ & $r=0.98$ & $r=0.78$ & $r=0.77$ \\
\hline LVEF (\%) & $69.5 \pm 10.6$ & $69.5 \pm 11.0$ & $64.8 \pm 11.6$ & $r=0.80$ & $r=0.60$ & $r=0.65$ \\
\hline \multicolumn{7}{|l|}{ QGS (\%) } \\
\hline All LVEF & $65.4 \pm 13.8$ & $68.4 \pm 15.2$ & $64.8 \pm 11.6$ & $P=0.0002$ & NS & $P=0.019$ \\
\hline Small-heart LVEF & $75.0 \pm 9.6$ & $79.5 \pm 8.3$ & $70.1 \pm 6.8$ & $P=0.0005$ & $P=0.0076$ & $P<0.0001$ \\
\hline \multicolumn{7}{|l|}{ CardioREPO (\%) } \\
\hline All LVEF & $69.5 \pm 10.6$ & $69.5 \pm 11.0$ & $64.8 \pm 11.6$ & NS & $P<0.0001$ & $P<0.0001$ \\
\hline Small-heart LVEF & $72.3 \pm 9.0$ & $74.3 \pm 8.3$ & $70.1 \pm 6.8$ & NS & NS & $P=0.0068$ \\
\hline
\end{tabular}

Conventional = conventional SPECT; echo = echocardiography; IQ = IQ•SPECT; NS = not statistically significant.

Data are mean \pm SD.

SPECT and IQ•SPECT when calculated using QGS $(P=$ $0.0002)$, there was no significant difference when calculated using cardioREPO.

Myocardial perfusion images showing normal findings on conventional SPECT and IQ•SPECT with and without attenuation and scatter correction are presented in Figure 4. The IQ•SPECT images with correction show an apparent perfusion defect at the anterior apex, but aside from that, the myocardial walls are more uniform than in uncorrected images. In Figure 5, a perfusion defect at the inferolateral wall is evident. Perfusion defects at the inferior and inferolateral walls are difficult to diagnose using uncorrected IQ•SPECT because the images can lack homogeneity and show false-positive defects at this location. However, correction is able to decrease body-attenuation artifacts at this location.

\section{DISCUSSION}

Compared with conventional SPECT, IQ•SPECT tends to overestimate LVEF, particularly in small-heart patients.
According to our data, the difference in LVEF between IQ•SPECT and conventional SPECT in small-heart patients is less when calculated using cardioREPO than when calculated using QGS.

\section{IQ·SPECT Artifacts}

In our experience, although the acquisition may be shorter for IQ•SPECT than conventional SPECT, the IQ•SPECT images may show distortion and unexpected artifacts caused by inappropriate positioning, body motion, body attenuation, and the characteristics of the SMARTZOOM collimator.

Caobelli et al. reported that inappropriate positioning of the heart affects the diagnostic accuracy of IQ•SPECT and that images of lesser quality can show inhomogeneity and false defects in the inferolateral walls $(18,19)$. Therefore, correct positioning of the heart is important in IQ•SPECT. However, occasionally it may be difficult to center the heart in the "sweet spot," the region of highest magnification of the multifocal collimators, especially for unskilled operators.
FIGURE 3. Bland-Altman analysis of LVEF on conventional SPECT with QGS vs. IQ·SPECT with QGS (A); conventional SPECT with QGS vs. echocardiography (B); IQ.SPECT with QGS vs. echocardiography (C); conventional SPECT with cardioREPO vs. IQ.SPECT with cardioREPO (D); conventional SPECT with cardioREPO vs. echocardiography (E); and IQ·SPECT with cardioREPO vs. echocardiography (F). Gray lines are mean \pm 1.96 SD. Black lines are linear regression lines.
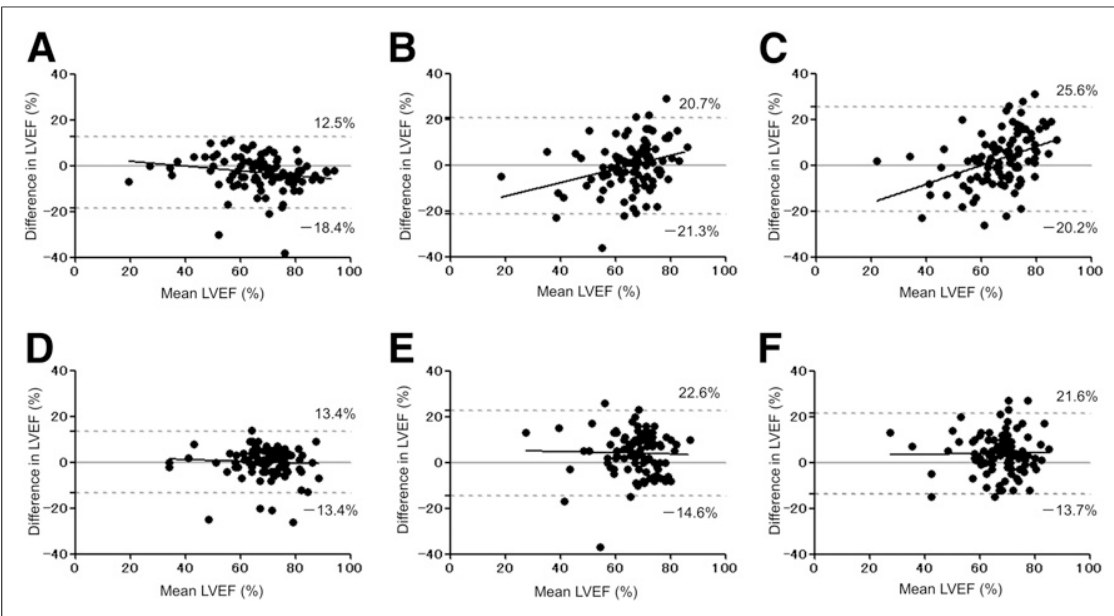


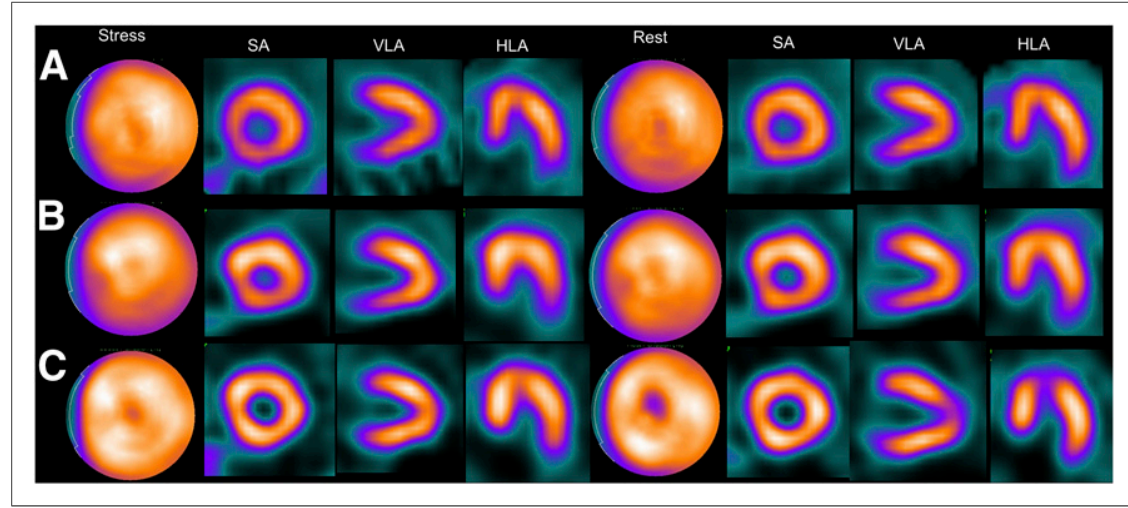

FIGURE 4. Myocardial perfusion SPECT images showing normal findings in 85-y-old man. (A) Conventional SPECT. (B) IQ•SPECT without attenuation or scatter correction. (C) IQ•SPECT with attenuation and scatter correction. HLA = horizontal long axis; $\mathrm{SA}=$ short axis; $\mathrm{VLA}=$ vertical long axis.
IQ•SPECT with attenuation correction frequently increases the hepatic count, which results in artifacts due to high extracardiac activity. Additionally, body motion tends to result in misdiagnosis when the patient is not properly restrained; as such, reacquisition should be considered after the linogram or sinogram has been checked.

\section{Acquisition Optimization}

Havel et al. reported that the average LVEF was $54.05 \% \pm$ $13.99 \%$ for IQ•SPECT (1 subset; 30 iterations; 14-mm FWHM gaussian filter) versus $61.93 \% \pm 12.24 \%$ for conventional SPECT (11). However, in that study the average ESV was $64.54 \pm 58.71$ for IQ•SPECT versus $50.32 \pm 59.94$ for conventional SPECT, values that are higher than in our study. Joergensen et al. reported that the average LVEF was $67.5 \% \pm$ $13.4 \%, 68.6 \% \pm 11.4 \%$, and $64.4 \% \pm 12.1 \%$ for, respectively, IQ•SPECT with 2 subsets, 15 iterations, and a 10-mm FWHM gaussian filter; IQ•SPECT with 1 subset, 12 iterations, and a 10-mm FWHM gaussian filter; and multigated blood-pool imaging (12). In that study, the average ESV was $33.3 \pm 40.7$ for IQ•SPECT with 2 subsets, 15 iterations, and a 10-mm FWHM gaussian filter versus $29.3 \pm 40.0$ for IQ•SPECT with 1 subset, 12 iterations and a 10-mm FWHM gaussian filter, values that are similar to those in our study.

One study found that a small heart occurs in $74 \%$ of Japanese women and $13 \%$ of Japanese men (20). In another study, the mean normal values and range (calculated by mean \pm 2 SDs) for ESV using ${ }^{99 m}$ Tc-sestamibi/tetrofosmin myocardial perfusion imaging (16-frame gated study) were $29 \mathrm{~mL}$ (range, 10-47 mL) for men and $18 \mathrm{~mL}$ (range, 6$30 \mathrm{~mL}$ ) for women using QGS, and $28 \mathrm{~mL}$ (range, 16$40 \mathrm{~mL}$ ) for men and $20 \mathrm{~mL}$ (range, 11-29 $\mathrm{mL}$ ) for women using cardioREPO (21).

Japanese researchers have reported optimal protocols for IQ•SPECT. Kenda et al. reported that the optimal reconstruction parameters in ordered-subset conjugate gradient

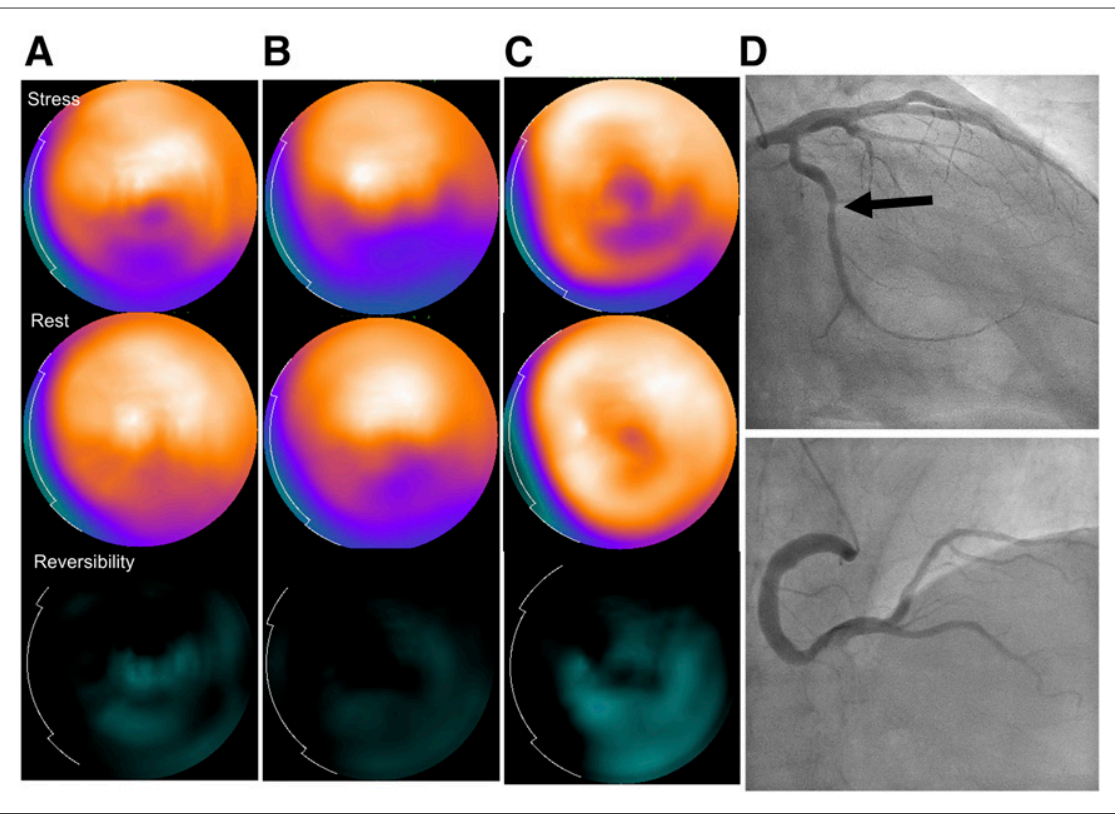

FIGURE 5. Myocardial perfusion SPECT images showing perfusion defect in inferolateral wall of 66-y-old man. (A) Conventional SPECT. (B) IQ•SPECT without attenuation or scatter correction. (C) IQ•SPECT with attenuation and scatter correction. (D) Invasive coronary angiography showing stenosis of left circumflex coronary artery (arrow), supporting the SPECT findings. 
minimization for IQ•SPECT are 1 subset and 30 iterations; for these parameters, the left ventricular volume was similar to the true volume (22). They conducted the evaluation using an RH-2 cardiac phantom (Kyoto Kagaku Co., Ltd.) containing a solution of ${ }^{99 \mathrm{~m}} \mathrm{Tc}$. However, their verification using a large-volume phantom was not as accurate as a study targeting small-heart patients.

Matsutomo et al. reported that EDV, ESV, and LVEF did not significantly differ between IQ•SPECT (1 subset; 30 iterations; 13-mm FWHM gaussian filter) and conventional SPECT with a ${ }^{99 \mathrm{~m}}$ Tc-labeled agent (13). Although the mean LVEF in that study tended to be higher for IQ•SPECT than for conventional SPECT, the difference was not significant $(68.3 \% \pm 12.1 \%$ vs. $64.8 \% \pm 11.8 \% ; P=0.269)$. The study was limited by a small number of patients, 25 , with only 7 being female, and the small-heart effect of the IQ•SPECT system was not clarified.

\section{Comparison of QGS and CardioREPO}

A working group of the Japanese Society of Nuclear Medicine has created databases of normal values for Japanese LVEF. The mean normal values and range (calculated by

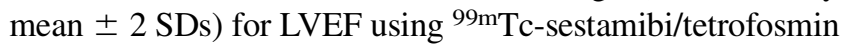
myocardial perfusion imaging (16-frame gated study) were $67 \%$ (range, $54 \%-79 \%$ ) for men and $72 \%$ (range, 61\%-83\%) for women using QGS, and 72\% (range, 61\%-83\%) for men and $75 \%$ (range, 64\%-85) for women using cardioREPO (21). In our overall results using QGS, the measurement of LVEF was significantly elevated and overestimated with IQ•SPECT compared with conventional SPECT and echocardiography. The tendency was significant in small-heart patients.

In small hearts, the true volume is underestimated, and this effect is higher for ESV than for EDV, resulting in an increase in LVEF. The small-heart effect, which has been seen in various studies $(6-10,16,23,24)$, is caused by optimization of the SPECT reconstruction method for a blurred myocardial wall. Nakajima et al. reported that quantification of gated SPECT for small hearts can be improved by using a high cutoff frequency for the SPECT filter, a high system resolution, and appropriate zooming and that improvement of spatial resolution can significantly decrease the small-heart effect (23). Additionally, cardioREPO, with specific algorithms compensating for an overestimated LVEF, has been more useful than QGS in addressing the small-heart effect $(6,16,24)$ and has adopted a corrected algorithm to minimize this effect, particularly in Japanese women and children $(21,24)$. In our study, LVEF for IQ•SPECT reconstructed using ordered-subset conjugate gradient minimization with 1 subset, 30 iterations, and a 9.6-mm FWHM gaussian filter was more similar to LVEF for conventional SPECT reconstructed using filtered backprojection with cardioREPO than to LVEF for conventional SPECT reconstructed using QGS.

\section{CONCLUSION}

IQ•SPECT outperforms conventional SPECT in producing images of hearts with small ventricular chambers. In addition, cardioREPO is designed to work with small hearts and outperforms QGS for this use.

\section{DISCLOSURE}

Kenichi Nakajima conducts collaborative research with FUJIFILM RI Pharma Co., Ltd., (Tokyo, Japan) on the development of software programs, and with Siemens (Erlangen, Germany) on the development of the IQ•SPECT system. This work was conducted partly as collaborative research with Siemens. No other potential conflict of interest relevant to this article was reported.

\section{ACKNOWLEDGMENTS}

We are grateful to Masaaki Kawamura, Syuhei Minami, Ayumi Morishita, Ayano Hosoki, Keita Sakuda, Haruka Nunome, Shinsuke Hanaoka, and all the radiologic technologists at Kanazawa University Hospital for providing technical support.

\section{REFERENCES}

1. Rajaram R, Bhattacharya M, Ding X, et al. Tomographic performance characteristics of the IQ-SPECT system. In: 2011 IEEE Nuclear Science Symposium Conference Record. Piscataway, NJ: IEEE; 2011:2451-2456.

2. Imbert L, Poussier S, Franken PR, et al. Compared performance of highsensitivity cameras dedicated to myocardial perfusion SPECT: a comprehensive analysis of phantom and human images. J Nucl Med. 2012;53: 1897-1903.

3. Lyon MC, Foster C, Ding X, et al. Dose reduction in half-time myocardial perfusion SPECT-CT with multifocal collimation. J Nucl Cardiol. 2016;23: 657-667.

4. Caobelli F, Thackeray JT, Soffientini A, Bengel FM, Pizzocaro C, Guerra UP. Feasibility of one-eighth time gated myocardial perfusion SPECT functional imaging using IQ-SPECT. Eur J Nucl Med Mol Imaging. 2015;42:19201928.

5. DePuey EG. Advances in SPECT camera software and hardware: currently available and new on the horizon. J Nucl Cardiol. 2012;19:551-581.

6. Nakajima K, Okuda K, Nystrom K, et al. Improved quantification of small hearts for gated myocardial perfusion imaging. Eur J Nucl Med Mol Imaging. 2013;40:11631170 .

7. Paul AK, Nabi HA. Gated myocardial perfusion SPECT: basic principles, technical aspects, and clinical applications. J Nucl Med Technol. 2004;32:179-187.

8. Hambye AS, Vervaet A, Dobbeleir A. Variability of left ventricular ejection fraction and volumes with quantitative gated SPECT: influence of algorithm, pixel size and reconstruction parameters in small and normal-sized hearts. Eur J Nucl Med Mol Imaging. 2004;31:1606-1613.

9. Khalil MM, Elgazzar A, Khalil W, Omar A, Ziada G. Assessment of left ventricular ejection fraction by four different methods using ${ }^{99 \mathrm{~m}} \mathrm{Tc}$ tetrofosmin gated SPECT in patients with small hearts: correlation with gated blood pool. Nucl Med Commun. 2005;26:885-893.

10. Danesh-Sani SH, Zakavi SR, Oskoueian L, Kakhki VR. Comparison between ${ }^{99 m}$ Tc-sestamibi gated myocardial perfusion SPECT and echocardiography in assessment of left ventricular volumes and ejection fraction-effect of perfusion defect and small heart. Nucl Med Rev Cent East Eur. 2014; 17:70-74.

11. Havel M, Kolacek M, Kaminek M, Dedek V, Kraft O, Sirucek P. Myocardial perfusion imaging parameters: IQ-SPECT and conventional SPET system comparison. Hell J Nucl Med. 2014;17:200-203.

12. Joergensen T, Hansson SH. Evaluation of the left ventricular ejection fraction with gated IQ-SPECT myocardial perfusion imaging. J Nucl Med Technol. 2015;43:193-200. 
13. Matsutomo N, Nagaki A, Sasaki M. Performance of myocardial perfusion imaging using multi-focal fan beam collimator with resolution recovery reconstruction in a comparison with conventional SPECT. Asia Ocean J Nucl Med Biol. 2014;2:111-119.

14. Matsuo S, Nakajima K, Onoguchi M, et al. Nuclear myocardial perfusion imaging using thallium-201 with a novel multifocal collimator SPECT/CT: IQSPECT versus conventional protocols in normal subjects. Ann Nucl Med. 2015;29:452-459.

15. Teichholz LE, Kreulen T, Herman MV, Gorlin R. Problems in echocardiographic volume determinations: echocardiographic-angiographic correlations in the presence or absence of asynergy. Am J Cardiol. 1976;37:7-11.

16. Kondo $\mathrm{C}$, Watanabe $\mathrm{E}$, Momose $\mathrm{M}$, et al. In vivo validation of gated myocardial SPECT imaging for quantification of small hearts: comparison with cardiac MRI. EJNMMI Res. 2016;6:9.

17. Lomsky M, Richter J, Johansson L, et al. A new automated method for analysis of gated-SPECT images based on a three-dimensional heart shaped model. Clin Physiol Funct Imaging. 2005;25:234-240.

18. Caobelli F, Ren Kaiser S, Thackeray JT, Bengel FM, Chieregato M. The importance of a correct positioning of the heart using IQ-SPECT system with multifocal collimators in myocardial perfusion imaging: a phantom study. $J$ Nucl Cardiol. 2015;22:57-65.
19. Caobelli F, Ren Kaiser S, Thackeray JT, et al. IQ SPECT allows a significant reduction in administered dose and acquisition time for myocardial perfusion imaging: evidence from a phantom study. J Nucl Med. 2014; 55:2064-2070.

20. Nakajima K, Kusuoka H, Nishimura S, Yamashina A, Nishimura T. Normal limits of ejection fraction and volumes determined by gated SPECT in clinically normal patients without cardiac events: a study based on the J-ACCESS database. Eur J Nucl Med Mol Imaging. 2007;34:1088-1096.

21. Nakajima K, Matsumoto N, Kasai T, Matsuo S, Kiso K, Okuda K. Normal values and standardization of parameters in nuclear cardiology: Japanese Society of Nuclear Medicine working group database. Ann Nucl Med. 2016;30:188-199.

22. Kenda S, Onishi H, Nakamoto K. Optimization of reconstruction parameters using a multi-focal fan beam collimator in myocardial perfusion single photon emission computed tomography study. Nihon Hoshasen Gijutsu Gakkai Zasshi. 2014;70:662-669.

23. Nakajima K, Taki J, Higuchi T, et al. Gated SPET quantification of small hearts: mathematical simulation and clinical application. Eur J Nucl Med. 2000;27: 1372-1379.

24. Yoneyama H, Nakajima K, Okuda K, et al. Reducing the small-heart effect in pediatric gated myocardial perfusion single-photon emission computed tomography. J Nucl Cardiol. May 19, 2016 [Epub ahead of print]. 